\title{
MICROVORTEX GENERATOR FOR SCRAMJET INLET APPLICATION
}

\author{
A. C. Idris ${ }^{1}$, M. R. Saad ${ }^{1}$, and K. Kontis ${ }^{2}$ \\ ${ }^{1}$ National Defence University of Malaysia (NDUM) \\ Sungai Besi Camp, Kuala Lumpur 57000, Malaysia \\ ${ }^{2}$ University of Glasgow \\ Glasgow G12 8QQ, Scotland, United Kingdom
}

\begin{abstract}
Global measurement approach in characterizing a generic scramjet inlet has enabled the investigation of various flow phenomena typically occurred on an inlet to be investigated further. This paper examines the effectiveness of microvortex generator (MVG) array in suppressing boundary layer separations on such an inlet. Global measurement of pressure and temperature were employed to visualize vortex pairs emanating from the MVG. The global pressure map showed the benefit of lower reattachment peak pressure that usually accompanies the large separation bubble at a scramjet inlet throat.
\end{abstract}

\section{INTRODUCTION}

Comprehensive global measurement of flow on a generic scramjet inlet-isolator has been demonstrated by Idris et al. [1]. They showed that it was possible to apply highly sensitive, platinum-based, pressure sensitive paint (PSP) onto the external and internal surface of a scramjet inlet at Mach 5 and produce amazing flow topology with rich quantitative information. Their techniques open up new opportunities to fully understand different complex flow phenomena that exist in a scramjet inlet. This paper aims to replicate their techniques in order to investigate the mechanism of flow separation control using micro vortex generator in scramjet inlet application.

In the case considered, the separation at the compression corner was observed between the first and second ramp and another at the inlet shoulder. Peculiarly, the reattachment along with the decay of streamwise vortices/Goertler vortices on the second ramp could help in ensuring turbulent boundary layer flowing into isolator section, thus preventing the separation at shoulder. Nonetheless, it has been reported by Reinartz et al. [2] and Nguyen et al. [3] that flow 'relaminarization' could occur due to pressure drop and acceleration of flow at the

(C) The Authors, published by EDP Sciences. This is an open access article distributed under the terms of the Creative Commons Attribution License 4.0 (http://creativecommons.org/licenses/by/4.0/). 
expansion corner (inlet shoulder). Following the example from Reinartz et al. [2], vortex generator is placed on the second ramp to control shoulder separation. Differently from their study, MVG array are utilized in this study to achieve the same effect. The MVG array was shaped from strip of sand paper and the effectiveness of three different grades of sand paper are discussed in the next section.

\section{METHODOLOGY AND EXPERIMENTAL SETUP}

\subsection{High Supersonic Wind Tunnel}

All experiments reported in this paper were conducted in the blowdown high supersonic wind tunnel (HSST) at The University of Manchester, United Kingdom, such as shown in Fig. 1. The tunnel operates using compressed air stored at 16 bar. The total pressure was set at $645 \mathrm{kPa}$ using the Type C4 model 208/3 dome valve.

Heating of the working fluid is provided by 24-kilowatt direct current electric resistive heater. The stagnation temperature is about $370 \mathrm{~K}$. The axisymmetric nozzle is changeable between Mach 4, 5, and 6. All experiments are conducted using Mach 5 nozzle as the generic scramjet inlet model is designed to operate at this flow speed.

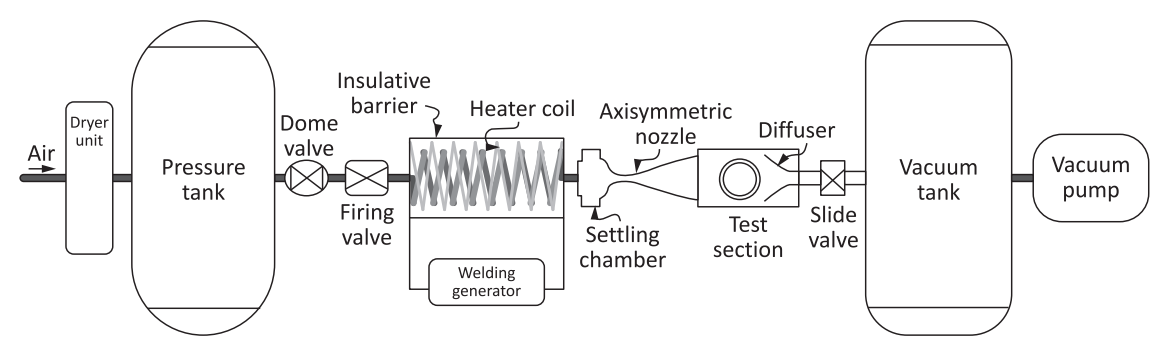

Figure 1 High supersonic tunnel at The University of Manchester

\subsection{Pressure Sensitive Paint Measurement}

Figure 2 shows the side view of PSP system setup optimized for measuring pressure intensity on compression and body-side isolator surface. The camera was placed on top of the test section, with its lens focused onto the model through the top window at an angle. The camera setup angle was not important because the model length was calibrated by markings on the model. The angle of camera 


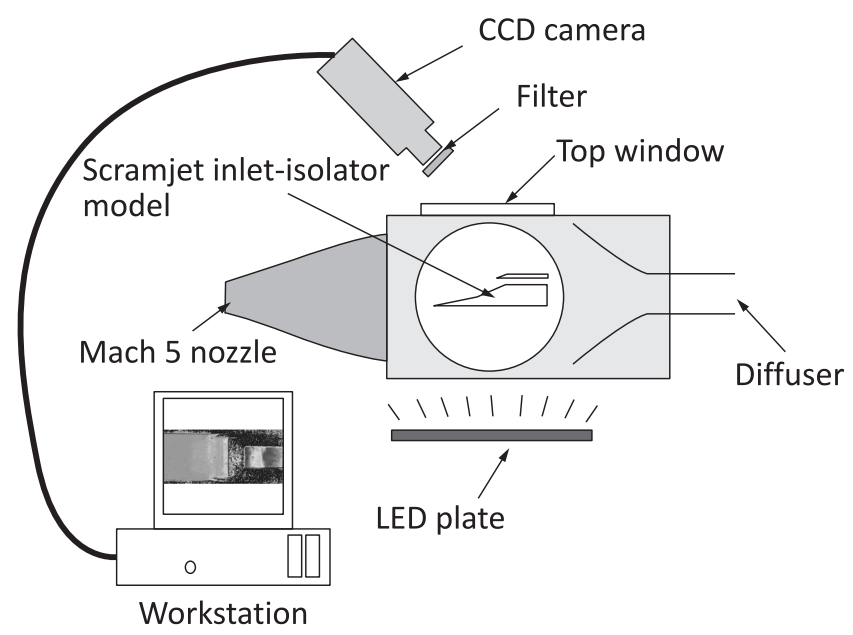

Figure 2 Side view of PSP system setup for compression ramps and isolator surface pressure measurement

placement was adjusted as necessary to accommodate the full model length. The scramjet inlet-isolator model was fixed on sting holder, which can be rotated on horizontal-vertical plane to vary the model angle-of-attack. For this setup, LED plates were placed on both side windows for even illumination on the model. LED lamps were preferred than other light source due to their monochromatic nature. A filter was used for the camera to minimize recording of unwanted photon from environment. For in situ calibration, static pressure distribution was measured using discreet transducer at the same time as pressure intensities were recorded. Intensity measurements were done in the darkest possible condition to reduce light contamination.

Scientific grade 12 bit CCD camera model LaVision Imager Intense was used as the main photon detector device for both setups. The camera was set to optimum exposure time of $7.5 \mathrm{~ms}$ with frame rate of $10 \mathrm{~Hz}$. Sufficient exposure time was needed to allow the camera to capture the maximum photons possible and make use of its full-well capacity. Recorded images were preprocessed and viewed in real time by using Davis 7.0 software installed on Microsoft Windowsbased workstation connected to the camera. MATLAB image processing toolbox was then utilized for further data processing.

\subsection{Infrared Measurement}

FLIR ThermaCAM SC 3000 Infrared (IR) camera was available for the current experiments. The images can be captured with frequency of up to $900 \mathrm{~Hz}$ in 14 - 


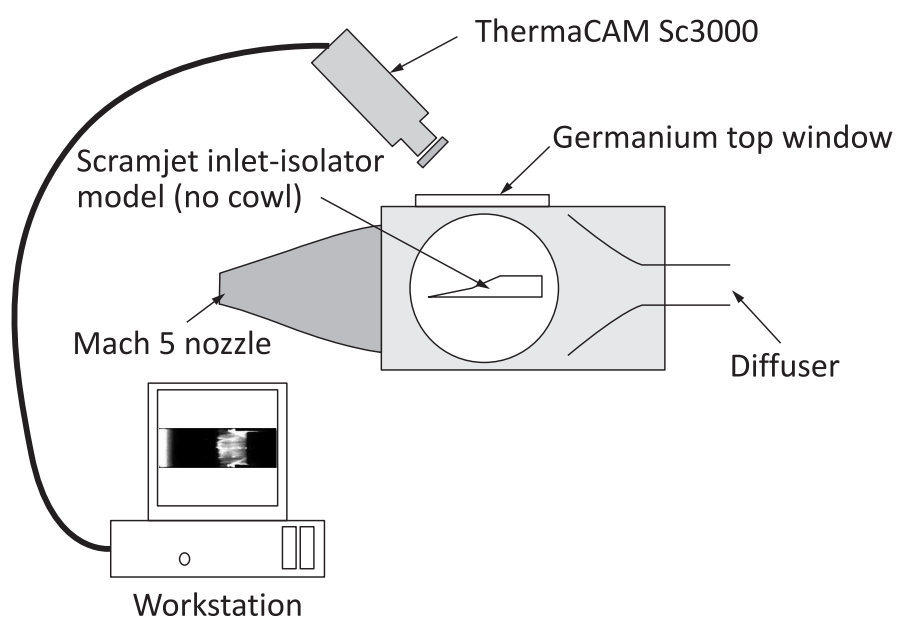

Figure 3 Schematic of IR thermography experimental setup

bit format. For current experiments, the camera was set to capture only $100 \mathrm{~Hz}$. Its thermal sensitivity was $20 \mathrm{mK}$ at standard temperature of $30{ }^{\circ} \mathrm{C}$. It can automatically correct for different atmospheric conditions based on distance, temperature, and relative humidity. ThermaCAM Researcher Software was used to process in real time the raw image captured by the camera.

The camera was placed on top of the test section similar to PSP setup. This was because only top plate can be fitted with germanium window required for IR measurement. The model was painted black to achieve emissivity of about 0.95 . As the material of scramjet inlet-isolator is made from aluminum, which has high heat conductivity, the measurement of the heat transfer would not be shown. Instead, vortex formation, if any, would be visualized by delta-temperature map taken every $2 \mathrm{~s}$.

The schematic of the experimental setup is shown in Fig. 3.

\section{ROUGHNESS-INDUCED TRANSITION FOR SUPPRESSING COMPRESSION CORNER SEPARATION}

An exploratory study of boundary layer trip using sand paper strip has been done on the compression ramp at Mach 5 with no angle-of-attack (AoA). Three sand paper strips of grid size P60, P100, and P150 have been selected for this study. They were cut into strip of size $5 \times 36 \mathrm{~mm}$. Their location on the first ramp was selected by considering the requirements of having low boundary layer edge 


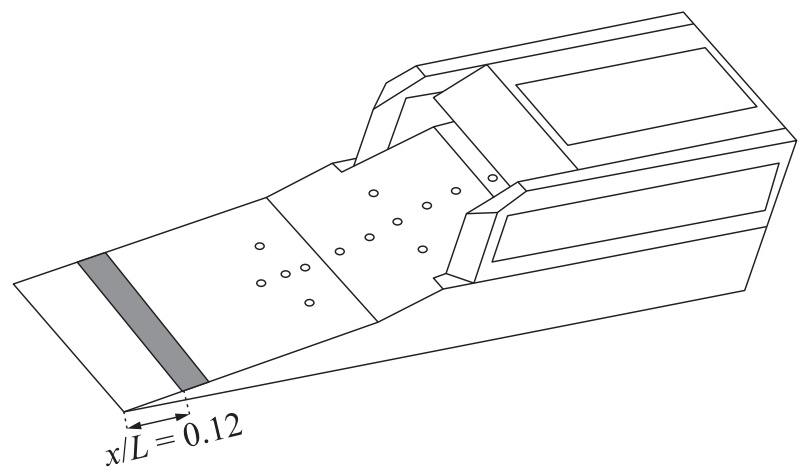

Figure 4 Position of sand-paper strip from the leading edge

Mach number and thick enough boundary layer to accommodate the strip [4]. The location selected is $x / L=0.12$ where the local boundary layer thickness was found to be of $0.7 \mathrm{~mm}$ (Fig. 4). The strip was pasted onto the aluminum surface using double-sided tape of thickness $0.08 \mathrm{~mm}$ where the leading edge of the roughness strip was aligned at the $x$-coordinate selected. The results for different cases are presented in Table 1.

Processed Schlieren images (not shown in this paper) indicate that P150-strip case was not able to affect the compression corner separation. The boundary layer edge was clearly visible up to the separation point. With P100-strip case, the flow transitioned from laminar to turbulence at $x / L=0.3$ while the flow still separates at the compression corner. The separation shock was barely visible and the reattachment shock moved closer to compression corner. Further increasing the roughness size in P60-strip case, the separation at compression corner was fully eliminated and the transition onset point moved further upstream $(x / L$ $=0.26)$.

Table 1 Location of transition starting point for different grades of sand paper

\begin{tabular}{lcccc}
\hline \multicolumn{1}{c}{ Case } & $\begin{array}{c}\text { Total strip } \\
\text { height, mm }\end{array}$ & Mach number & $\begin{array}{c}\text { Unite } \\
\text { Reynolds number, } \\
\mathrm{m}^{-1}\end{array}$ & $\begin{array}{c}\text { Transition } \\
\text { location } x / L\end{array}$ \\
\hline P150-strip & 0.28 & $5 \pm 0.4 \%$ & $13.2 \cdot 10^{6}$ & none \\
P100-strip & 0.43 & $5 \pm 0.4 \%$ & $13.2 \cdot 10^{6}$ & 0.30 \\
P60-strip & 0.65 & $5 \pm 0.4 \%$ & $13.2 \cdot 10^{6}$ & 0.26 \\
MVG-array & 0.65 & $5 \pm 0.4 \%$ & $13.2 \cdot 10^{6}$ & 0.23 \\
\hline
\end{tabular}




\section{MICROVORTEX GENERATOR INDUCED TRANSITION FOR SUPPRESSING COMPRESSION CORNER SEPARATION}

The effectiveness of P60-strip case in eliminating compression separation has made it as a suitable candidate for exploratory study on MVG in scramjet inlet application. The P60 sand paper strip has been cut into a vortex generator array of three connected triangular vortex generator. Each single vortex generator has spanwise length of $7 \mathrm{~mm}$ and streamwise length of $5 \mathrm{~mm}$ such as shown in Fig. 5 . This corresponds to hypotenuse length of $c=6.1 \mathrm{~mm}$ for each single MVG. The half apex angle was set to be $A=35^{\circ}$. The height of the vortex generator is similar to P60-strip element thickness of $h=0.65 \mathrm{~mm}$. The dimension was chosen for purely convenient reason, which does not place too much importance in optimum shape of the MVG. This was due to this experiment being exploratory in nature; thus, any possibility in optimization was reserved for future studies. Three single MVGs were positioned together into an array with distance between every apex set to $s=7 \mathrm{~mm}$ (Fig. 6). Its shape is similar to prism-type used in the article by Schulein and Trofimov [5]. Its spanwise location was set by aligning the centerline of the MVG array to coincide with the scramjet model centerline. The $x$-coordinate of the MVG array was then fixed at location $x / L=0.11$ from the inlet leading edge (Fig. 7).

Processed Schlieren images (not shown in this paper) indicate the effectiveness of the MVG in eliminating the compression corner separation. The transition region for MVG case was more upstream or at least comparable to that for P60-strip case (see Table 1). Beside the formation of turbulence, the MVG also promoted the formation of horseshoe vortex (Fig. 8) that may contribute to the elimination of compression corner separation. The MVG array achieved similar capability of eliminating separation at compression corner while at the same time having lower overall drag than P60 sand paper strip case.

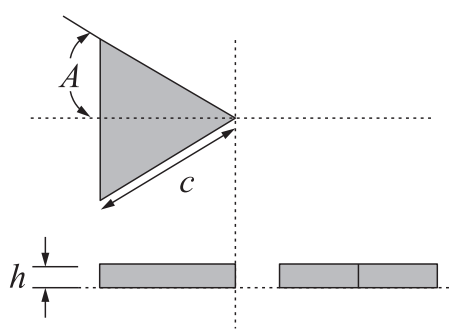

Figure 5 Dimension of a single MVG

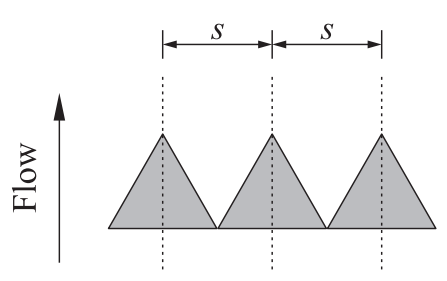

Figure 6 Distance between MVGs in an array 


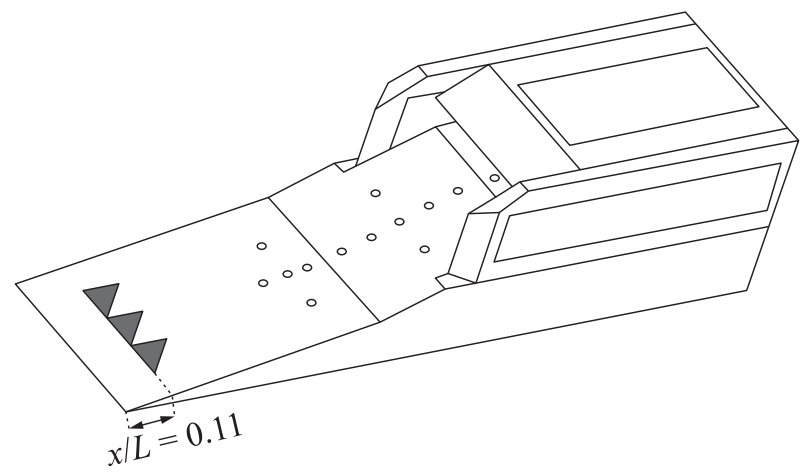

Figure 7 The MVG array was positioned at a distance $x / L=0.11$ from the leading edge and its middle apex coincide with inlet centerline

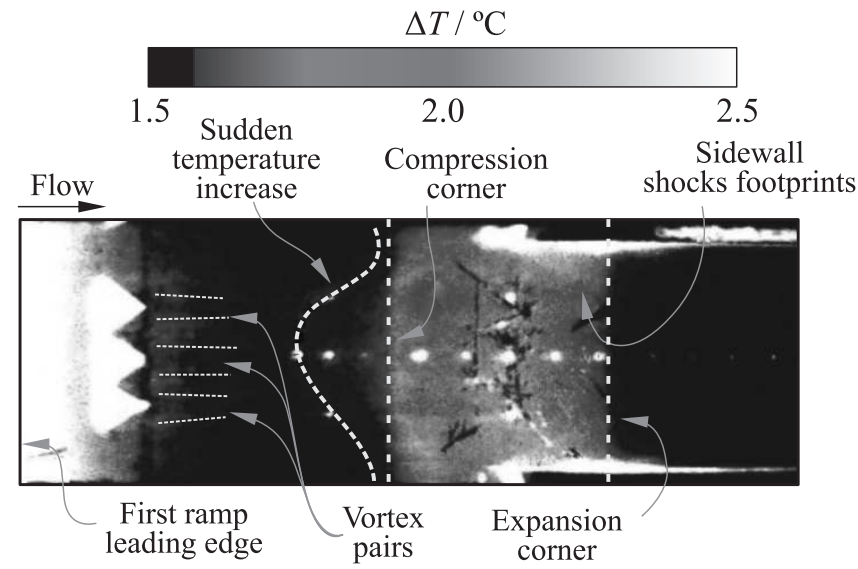

Figure 8 Horse-shoe vortex pair created by each MVG in the array as shown by delta-temperature map from IR thermography

\section{MICROVORTEX GENERATOR INDUCED TRANSITION FOR SUPPRESSING EXPANSION CORNER SEPARATION}

Eventhough the MVG-array has been able to control compression corner separation by inducing turbulent early on the first ramp, expansion corner relaminarization at shoulder means that boundary layer separation could still occur. With that in mind, the MVG array has been repositioned to the middle of the 


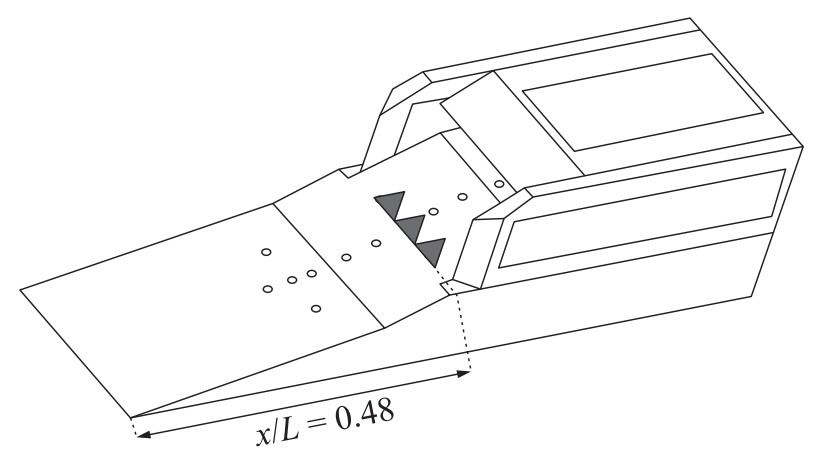

Figure 9 The MVG array was positioned at a distance $x / L=0.48$ from the leading edge and its middle apex coincides with inlet centerline

second ramp to test its effect in suppressing shoulder separation. Reinartz et al. [2] have demonstrated the way to minimize the extent of shoulder separation by placing a boundary layer trip device on the compression surface closest to the expansion corner without detail quantitative analysis. In this current study, the PSP technique has been applied to quantitatively characterize the isolator flow in the present MVG array. The MVG array was fixed just downstream of the reattachment point of the corner separation, at location $x / L=0.48$ (Fig. 9).

Figure 10 shows the comparison of isolator shock structures without (Fig. 10a) and with MVG array (Fig. 10b). Shoulder separation still occurs in Fig. $10 b$ but with a significantly reduced separation bubble height. Smaller shoulder boundary layer produced separation shock that was not strong enough to induce separation around the cowl tip region like in Fig. 10a. The separation induced by shoulder reattachment shock also appeared smaller in the MVG case. It was observed that the shock structures inside the isolator were faintly visible in the MVG-array case, indicating that the shocks were weaker in nature and that there was a high level of flow uniformity.

The pressure profiles taken on the centerline of the model for baseline and MVG case are compared in Fig. 11. The intensity measured by the PSP camera was calibrated against Kulite readings distributed on both the internal and external part of the scramjet inlet. Significant oscillations in pressure were detected for all transducer locations downstream of MVG location, which explains larger range of error bar. The PSP calibration curve for MVG-array case (not shown in this article) was satisfactory with coefficient $R^{2}=0.976$. Pressure predicted by the PSP method in unit bar was corrected to within \pm 0.048 (nondimensionalized unit).

In Fig. 11, one can see that in the isolator region, there was slight disagreement between discrete Kulite pressure transducer readings and PSP for no-MVG 


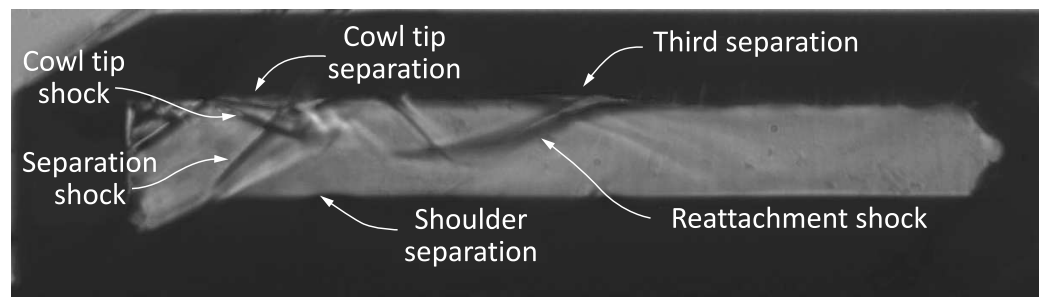

(a)

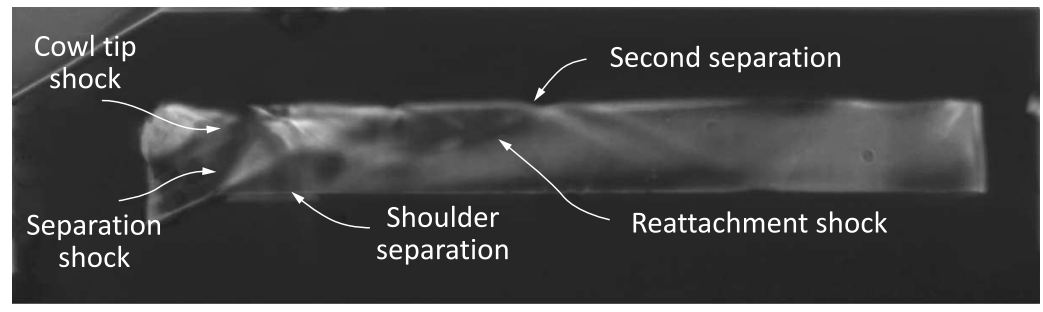

(b)

Figure 10 Experimental Schlieren image of isolator flow for baseline (without MVG) (a) and with MVG-array (b)

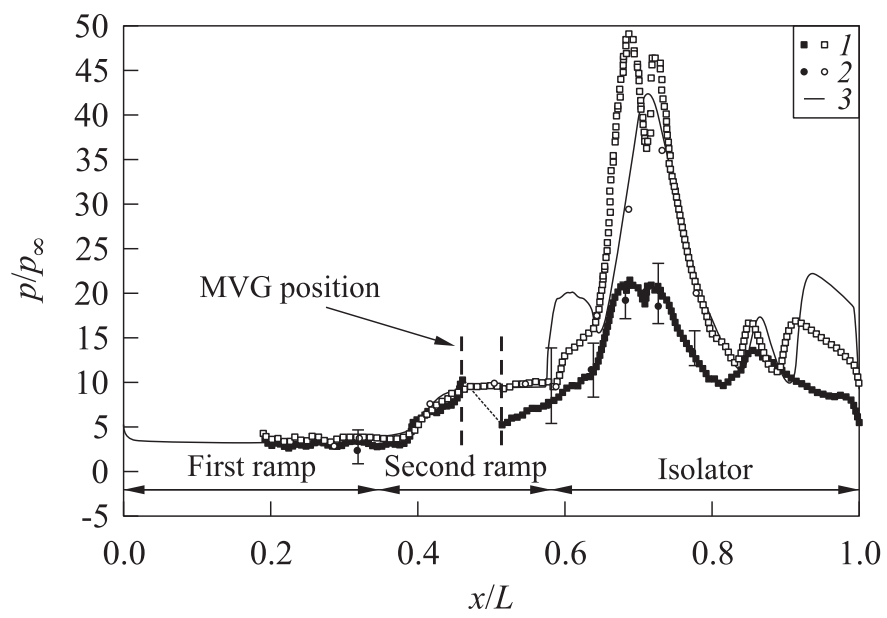

Figure 11 Normalized static pressure profile without (black signs) and with (filled signs) MVG: $1-\mathrm{PSP} ; 2-$ Kulite; and $3-\mathrm{SST}-k_{\omega}$ without MVG 
(baseline) case. However, fluid dynamic analysis using two-dimensional Shear Stress Transport (SST) $k_{\omega}$ model in FLUENT software was more in agreement with Kulite discrete readings.

The pressure measured using PSP for MVG-array case was consistently lower than the baseline case in the region downstream of MVG locations. Normally, the surface pressure was constant streamwise from the start to the end of the second ramp. By introducing the MVG-array in the middle of the second ramp, we could observe a drop of pressure just downstream of MVG-array position and before it a gradual increase.

Figure 11 also shows that even though separation still occurs inside the isolator, the severity of its reattachment shock has dropped by more than $50 \%$. The reattachment peak pressure for MVG-array case also moved upstream, closer to the shoulder. Subsequently, all pressure peaks inside the isolator have been reduced as well. This will results into higher flow uniformity exiting the isolator and improve the combustion process in a real scramjet engine.

The three-dimensional effect of MVG on the flow is shown in Fig. 12. On the second ramp surface, the pressure drop is confined only at a small streak downstream of each individual vortex generator. Similarly, the reduction in reattachment pressure peak is also limited in the middle region which is under

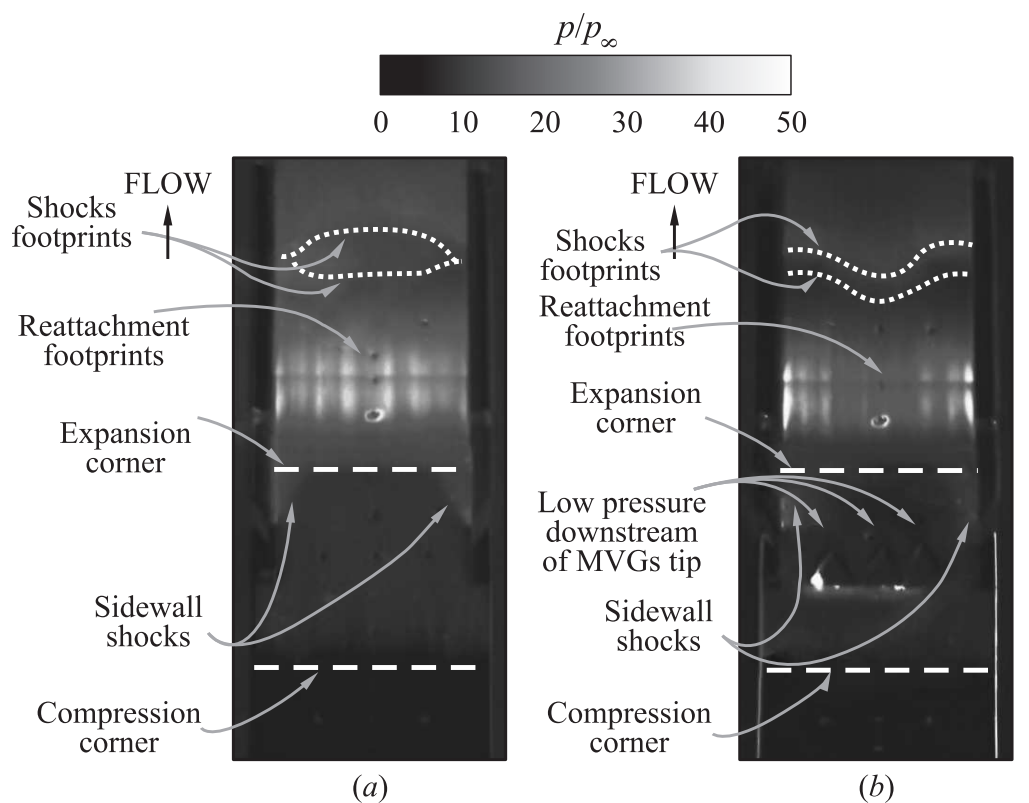

Figure 12 Inlet-isolator surface pressure comparison of no-MVG $(a)$ and with MVG (b). Image is processed from the raw PSP data 


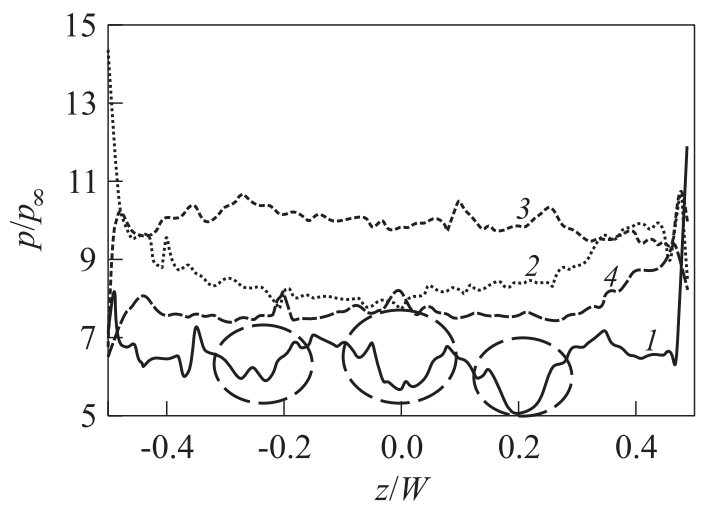

Figure 13 Spanwise pressure at different streamwise locations for the case with MVG. Plot is processed from the raw PSP data: $1-x / L=0.54 ; 2-0.59 ; 3-0.78$; and $4-x / L=0.99$

the influence of MVG, therefore resulting in the distortion of subsequent shock footprints further downstream. The shock footprints close to the isolator exit have been moved forward. This is due to the reduction of the expansion corner separation bubble size with the biggest reduction in size occurring at the middle of the isolator.

Spanwise pressure has been taken at the start $(x / L=0.59)$, middle $(x / L$ $=0.78)$, and end of isolator segment $(x / L=0.99)$ and compared in Fig. 13. The spanwise pressure at the position immediately downstream of MVG array $(x / L=0.54)$ has been included as well. The spanwise pressure was quite uniform from the start to the end. This is in contrast with the large fluctuation in spanwise pressure recorded at a location just downstream of MVG. Three trough in spanwise pressure can be identified (marked with dashed circles in Fig. 13) at the downstream of MVG. The spanwise location (i. e., in $z$-direction, normalized to isolator width) of the trough coincided well with the spanwise location of the three individual MVG tips. The drop in pressure proves the formation of three vortices emanating from each MVG tip before it decays. This is similar to the observation made by $\mathrm{Li}$ and $\mathrm{Liu}[6]$. The vortices were not detected inside the isolator and the resolution of PSP was not satisfactory to exactly pinpoint the location of their decay.

\section{CONCLUDING REMARKS}

The MVG has proven an effective countermeasure for mitigating boundary-layer separation. The mechanism is activated by a horseshoe vortex formation that 
flows from downstream of the MVG. In scramjet inlet application, the elimination of separation at shoulder improved the overall flow of the inlet. The structure of the inlet undergoes a much less impinging pressure from the boundary layer reattachment always linked to the separation.

\section{REFERENCES}

1. Idris, A. C., M. R. Saad, H. Zare-Behtash, and K. Kontis. 2014. Luminescent measurement systems for the investigation of a scramjet inlet-isolator. Sensors 14:66066632 .

2. Reinartz, B. U., C. D. Herrmann, J. Ballmann, and W. W. Koschel. 2003. Aerodynamic performance analysis of a hypersonic inlet isolator using computation and experiment. J. Propul. Power 19:868-875.

3. Nguyen, T., M. Behr, B. Reinartz, O. Hohn, and A. Gülhan. 2013. Effects of sidewall compression and relaminarization in a scramjet inlet. J. Propul. Power 29:628-638.

4. Berry, S.A., A.H. Auslender, A.D. Dilley, and J.F. Calleja. 2001. Hypersonic boundary-layer trip development for Hyper-X. J. Spacecraft Rockets 38:853-864.

5. Schülein, E., and V. M. Trofimov. 2011. Steady longitudinal vortices in supersonic turbulent separated flows. J. Fluid Mech. 672:451-476.

6. Li, Q., and C. Liu. 2010. Numerical investigations on the effects of the declining angle of the trailing-edge of MVG. AIAA Paper No. 2010-714. 